\title{
Curvas de crescimento em vacas de corte de diferentes tipos biológicos
}

\author{
Fabiane de Lima Silva(1), Maurício Mello de Alencar(2), Alfredo Ribeiro de Freitas ${ }^{(3)}$, \\ Irineu Umberto Packer ${ }^{(4)}$ e Gerson Barreto Mourão(5)
}

\begin{abstract}
(1)Universidade de São Paulo (USP), Escola Superior de Agricultura Luiz de Queiroz (Esalq), Programa de Pós-graduação em Ciência Animal e Pastagens, Avenida Pádua Dias, no 11, CEP 13418-900 Piracicaba, SP. E-mail: fabianezte@yahoo.com.br (2)Embrapa Pecuária Sudeste, Rodovia Washington Luiz, Km 234, CEP 13560-970 São Carlos, SP. E-mail: mauricio@cpsse.embrapa.br (3)Universidade Federal de São Carlos, Rodovia Washington Luiz, Km 235, CEP 13565-905 São Carlos, SP. E-mail: alfribeiro@hotmail.com ${ }^{(4)}$ In memoriam. ${ }^{(5)}$ USP, Esalq, Departamento de Zootecnia. E-mail: gbmourao@esalq.usp.br
\end{abstract}

Resumo - O objetivo deste trabalho foi selecionar o modelo de curvas de crescimento mais adequado e avaliar a influência de efeitos de ambiente e de grupo genético sobre os parâmetros estimados do modelo. Cinco modelos não lineares, Brody, Gompertz, Logístico, Von Bertalanffy e Richards, foram ajustados a dados de peso-idade coletados de 316 vacas, de quatro grupos genéticos: G (Nelore, 1/2Canchim $+1 / 2$ Nelore, $1 / 2$ Angus $+1 / 2$ Nelore e $1 / 2$ Simental $+1 / 2$ Nelore), do nascimento até 100 meses de idade; em duas estações do ano: E (primavera e outono). As vacas foram submetidas a dois níveis de concentrado (S) durante quatro meses, pós-desmama. $\mathrm{O}$ ajuste dos modelos foi realizado por mínimos quadrados ordinários, usando os pesos ponderado e não ponderado pelo inverso da variância. Os modelos Brody e Von Bertalanffy convergiram para todos os grupos genéticos; porém, o Brody foi o mais adequado. As estimativas do peso assintótico (A) e da taxa de maturação (k) do modelo Brody ponderado pelo inverso da variância foram analisadas por modelo misto, que incluiu efeito médio global e efeitos principais de G, E e S, e suas interações. O parâmetro A foi influenciado pelo efeito de $\mathrm{G}$ e $\mathrm{E}$, enquanto $\mathrm{k}$ foi influenciado por $\mathrm{S}$, o que indica que melhorias no manejo alimentar resultam em menor variação na forma das curvas de crescimento e em altas taxas de maturação.

Termos para indexação: cruzamento, curvas de crescimento, heterocedasticidade, modelo Brody, suplementação alimentar, variância ponderada.

\section{Growth curves in beef cows of different biological types}

Abstract - The objective of this study was to select the best growth curve model, and to evaluate the influence of environmental and genetic group effects on the estimated parameters of the model. Five nonlinear models, Brody, Gompertz, Logistic, Von Bertalanffy and Richards, were adjusted to age-weight data measured in 316 cows, obtained from four genetic groups: $G$ (Nellore, $1 / 2$ Canchim $+1 / 2$ Nellore, $1 / 2$ Angus $+1 / 2$ Nellore, and $1 / 2$ Simmental $+1 / 2$ Nellore), from birth to 100 months of age; in two calving seasons: $\mathrm{E}$ (spring and fall). The cows were submitted to two levels of concentrate (S) during four months, after weaning. The models were adjusted by ordinary least squares using inverse-variance weighted and unweighted mass. The Brody and Von Bertalanffy models converged for all genetic groups; however, the Brody model had a better fit. Estimates for asymptotic weight $(\mathrm{A})$ and maturing rate $(\mathrm{k})$ of the weighted Brody model were analyzed by a mixed model, which included overall effect and fixed effects of G, E, and S, and respective interactions. Parameter A was influenced by the effects of $\mathrm{G}$ and $\mathrm{E}$, while $\mathrm{k}$ was influenced by $\mathrm{S}$, indicating that improvements in feeding management result in less variation in the shape of growth curves and in increased rates of maturity.

Index terms: crossbreeding, growth curves, heteroscedasticity, Brody model, feed supplementation, weighted variance.

\section{Introdução}

O crescimento corporal da maioria das espécies pode ser descrito por uma curva sigmoide e, portanto, ajustado por modelos não lineares, também conhecidos como regressões não lineares. Esses modelos, quando ajustados aos dados de peso, altura, comprimento e outras características quantitativas de interesse, ao longo do tempo, tanto em animais quanto em vegetais, permitem sintetizar grande número de medidas e informações em apenas alguns parâmetros de interpretação biológica. Assim, o estudo dos parâmetros dessas curvas é fundamental para os programas de melhoramento genético que visam alterar a forma das curvas de

Pesq. agropec. bras., Brasília, v.46, n.3, p.262-271, mar. 2011 
crescimento dos animais, sendo imprescindível na análise bioeconômica para, por exemplo, estimar, após os modelos ajustados, o tempo (idade) ótimo para o abate do animal, ou seja, o momento em que a curva de crescimento médio dos animais atinge o seu máximo, ou seja, a maturidade (A). Após este ponto, o consumo é destinado à manutenção do animal, a conversão alimentar é baixa e o custo para manter o animal a campo ou em confinamento é alto, o que indica o momento de abate ou descarte. O interesse pela aplicação dos modelos não lineares na área animal tem sido crescente nos últimos anos, como mostrado em diversos trabalhos (Brown et al., 1972b, 1976; Nobre et al., 1987; Perotto et al., 1992; Elias, 1998; Oliveira et al., 2000; Tedeschi et al., 2000; Paz et al., 2004; Freitas, 2005; Forni et al., 2007), principalmente com os modelos Brody, Von Bertalanffy, Logístico, Gompertz e Richards.

A escolha do modelo mais adequado para estimar o crescimento em função da idade é dependente, entre outros fatores, da raça, do ambiente, da idade do animal nas últimas pesagens e do modelo propriamente dito.

Embora haja trabalhos com diversas raças bovinas, há poucas informações sobre as condições brasileiras referentes às curvas de crescimento de vacas cruzadas até a idade adulta, pois, frequentemente, os ajustes dos modelos têm sido realizados com conjuntos de dados que não contemplam o crescimento até a maturidade, $o$ que prejudica as inferências obtidas dos parâmetros.

No estudo de curvas de crescimento, geralmente ocorre heterogeneidade das variâncias entre as pesagens avaliadas no animal, já que a variância dos pesos corporais aumenta à medida que aumenta a idade. Entretanto, esse fato não é levado em consideração na maioria dos estudos de curvas de crescimento. A heterogeneidade das variâncias pode ser considerada como o resultado de "distúrbios de regressão", pois os dados são tomados longitudinalmente em cada animal (Pasternak \& Shalev, 1994; Guedes et al., 2004; Mazzini et al., 2005; Mendes et al., 2008). Quando a pressuposição de homogeneidade de variância é ignorada, podem ocorrer estimativas viesadas dos parâmetros, como a super ou subestimação das variâncias (Souza, 1998).

Uma alternativa para solucionar esse problema é o uso de regressão não linear ponderada, com o inverso da variância dos pesos nas diferentes classes de idade, para reduzir a heterogeneidade das variâncias dos pesos ao longo do tempo (Pasternak \& Shalev, 1994). Estes autores estimaram os parâmetros do modelo Gompertz para dados simulados de pesos de frango de corte, com uso de regressão não linear simples e ponderada, cuja maior eficiência foi verificada pelo inverso das variâncias dos pesos nas diferentes idades.

Elias (1998) constatou, ao comparar modelos não lineares de maneira não ponderada e ponderada pelo inverso da variância no ajuste de pesos de vacas zebuínas, que a ponderação melhorou a qualidade dos ajustes e reduziu o erro das estimativas dos parâmetros.

O objetivo deste trabalho foi selecionar o modelo de curvas de crescimento mais adequado e avaliar a influência de efeitos de ambiente e de grupo genético sobre os parâmetros estimados do modelo.

\section{Material e Métodos}

Foram utilizados 3.523 dados de peso-idade de 316 fêmeas bovinas, do nascimento até 100 meses de idade, nascidas entre 1998 e 2001, em duas estações de nascimento, pertencentes aos grupos genéticos: Nelore (NEL, $\mathrm{n}=86$ ), $1 / 2$ Canchim $+1 / 2$ Nelore $(\mathrm{CN}, \mathrm{n}=87), 1 / 2$ Angus $+1 / 2$ Nelore $(\mathrm{AN}, \mathrm{n}=73) \mathrm{e}$ $1 / 2$ Simental $+1 / 2$ Nelore $(\mathrm{SN}, \mathrm{n}=70$ ), desenvolvidos na Embrapa Pecuária Sudeste, no Município de São Carlos, SP (22 $011^{\prime} \mathrm{S}, 4^{\circ} 54^{\prime} \mathrm{W}$ e $836 \mathrm{~m}$ de altitude), sob clima tropical de altitude. Os animais eram mantidos em pastagem de capim-coastcross [Cynodon dactylon (L.) Pers cv. Coastcross], em Latossolo Vermelho distroférrico típico.

As fêmeas destes quatro grupos genéticos, nascidas no outono (abril a junho) de 1998, 1999 e 2000, receberam, após a desmama (novembro-dezembro), dois níveis de suplementação (S): 0 e $3,0 \mathrm{~kg}$ de concentrado por animal por dia, durante quatro meses. Os animais foram distribuídos em 16 piquetes, de $3.700 \mathrm{~m}^{2}$ cada, de capim-coastcross, com duas repetições e quatro bezerras por piquete. Os piquetes foram divididos por cerca elétrica (dois fios) e subdivididos com fio eletrificado em cinco piquetes menores de $740 \mathrm{~m}^{2}$ cada, para utilização em pastejo rotacionado, com adubação de cobertura com NPK. O pastejo foi, em média, de cinco dias e o período de descanso de 20 dias. De dezembro a abril de cada ano, logo após a saída dos animais, foram aplicados $20 \mathrm{~kg}$ de adubo da fórmula NPK (20-05-20) em 
cada piquete, o que correspondeu a uma adubação de aproximadamente $300 \mathrm{~kg} \mathrm{ha}^{-1}$ por ano. O período de pastejo em todos os anos ocorreu de dezembro (início da fase pré-experimental) a maio. Durante o período da seca seguinte, os animais foram suplementados com cana-de-açúcar, ureia e 1,5 kg do mesmo concentrado. Esse manejo foi feito até que as novilhas atingissem a puberdade, caracterizada pela apresentação de dois cios com intervalo normal (17 a 25 dias).

Após a desmama (junho-julho) em 2001 e 2002, as fêmeas nascidas na primavera (setembro a dezembro) foram distribuídas em oito tratamentos com arranjo fatorial 4x2 (quatro grupos genéticos: NEL, CN, AN e SN, e dois níveis de suplementação: 1,5 e $3,0 \mathrm{~kg}$ de concentrado por animal por dia). Os animais foram mantidos nos mesmos piquetes utilizados para as fêmeas nascidas no outono e suplementados com cana-de-açúcar à vontade; este manejo foi utilizado até a entrada das fêmeas em reprodução. Para maiores detalhes sobre o manejo, veja Rodrigues et al. (2004).

Cada animal foi pesado nas seguintes épocas: ao nascer, aos seis meses, à desmama, ao ano, aos $18 \mathrm{e}$ 24 meses, em sua fase reprodutiva, à entrada e à saída da estação de monta, à parição e à desmama dos bezerros. Foram considerados dados de pesos de animais com pelo menos nove pesagens.

Para estimar o crescimento dos animais em função da idade, cinco modelos não lineares (Tabela 1) foram ajustados ao conjunto de dados de peso-idade das fêmeas. Nos modelos não lineares, yt representa o peso do animal em uma determinada idade; $t$, expressa dias ou meses; A é o peso assintótico do animal, ou seja, o peso adulto quando $\mathrm{t} \rightarrow \infty$; $\mathrm{k}$ mede a variação na velocidade do crescimento e está associado à taxa de maturidade, sendo que, em uma amostra ou população de animais, valores menores de $\mathrm{k}$ indicam animal de crescimento lento; b é a constante de integração, cuja função é modelar a forma da curva de crescimento a partir do nascimento para um formato sigmoidal; porém, não tem interpretação biológica; $m$ é uma constante do modelo de Richards, sendo determinante na origem das outras funções não lineares.

Para o ajuste, foi utilizado o procedimento NLIN do SAS, e as estimativas dos parâmetros foram obtidas por processo iterativo de Gauss-Newton modificado, desenvolvido por Hartley (1961), para modelos não lineares. O critério de convergência para as estimativas foi considerado quando:

$$
\left[\left(\mathrm{SQR}_{\mathrm{j}-1}-\mathrm{SQR}_{\mathrm{j}}\right) /\left(\mathrm{SQR}_{\mathrm{j}}+10^{-6}\right)\right] \leq 10^{-8},
$$

em que, $\mathrm{SQR}_{\mathrm{j}}$ é a soma de quadrado residual na j-ésima iteração.

Para verificar a influência da heterogeneidade de variância sobre as curvas de crescimento, considerouse a variância entre os pesos e o inverso dessa variância calculada nas várias classes de peso-idade, do nascimento até a idade adulta. No ajuste dos modelos, o inverso das variâncias foi utilizado como fator de ponderação pela opção Weight do procedimento NLIN do SAS (SAS Institute, 2004).

Os critérios utilizados para a seleção do modelo mais adequado foram: convergência ou não do modelo, coeficiente de determinação ajustado, quadrado médio do resíduo (QMR) e erro de predição médio (EPM). $\mathrm{O}$ coeficiente de determinação ajustado foi calculado por: $\mathrm{R}_{\text {ajustado }}^{2}=1$ - (SQR/SQTc), em que SQR é a soma de quadrados do resíduo e SQTc, a soma de quadrados total corrigida para o número de parâmetros do modelo. Contudo, o $\mathrm{R}_{\text {ajustado }}$ deve ser utilizado com cautela na avaliação da qualidade do ajuste, pois, no caso de modelos não lineares, a soma dos resíduos não é necessariamente igual à zero e a soma dos quadrados dos resíduos mais a soma dos quadrados da regressão não é necessariamente igual à soma total dos quadrados, o que pode afetar o coeficiente de determinação. O EPM foi obtido por meio da seguinte fórmula:

$$
\operatorname{EPM}=\sum_{\mathrm{i}=1}^{\mathrm{n}}\left(\mathrm{y}_{\mathrm{i}}-\hat{\mathrm{y}}_{\mathrm{i}}\right) / 100,
$$

em que y corresponde ao peso observado e $\hat{y}$ ao peso estimado nas $n$ pesagens. Valores positivos e negativos para EPM indicam, respectivamente, subestimativas e superestimativas do peso observado, enquanto sua magnitude mensura a aderência dos dados estimados aos dados observados.

Após a seleção do modelo mais adequado, as estimativas dos parâmetros A e $\mathrm{k}$ para cada animal foram analisadas pelo seguinte modelo:

Tabela 1. Forma geral dos modelos não lineares usados para o ajuste de curvas de crescimento, do nascimento até a idade adulta, de fêmeas bovinas de quatro grupos genéticos.

\begin{tabular}{lc}
\hline Modelo & Forma geral \\
\hline Brody & $\mathrm{Y}_{\mathrm{t}}=\mathrm{A}\left(1-\mathrm{Be}^{-\mathrm{kt}}\right)+\varepsilon$ \\
Von Bertalanffy & $\mathrm{Y}_{\mathrm{t}}=\mathrm{A}\left(1-\mathrm{Be}^{-\mathrm{kt}}\right)^{3}+\varepsilon$ \\
Richards & $\mathrm{Y}_{\mathrm{t}}=\mathrm{A}\left(1-\mathrm{Be}^{-\mathrm{kt}}\right)^{-\mathrm{m}}+\varepsilon$ \\
Logístico & $\mathrm{Y}_{\mathrm{t}}=\mathrm{A}\left(1-\mathrm{e}^{-\mathrm{kt}}\right)^{-\mathrm{m}}+\varepsilon$ \\
Gompertz & $\mathrm{Y}_{\mathrm{t}}=\mathrm{Ae}^{\mathrm{Be}(-\mathrm{kt})}+\varepsilon$ \\
\hline
\end{tabular}


$\mathrm{yijkl}=\mu+\mathrm{gi}+\mathrm{pj}+(\mathrm{gp}) \mathrm{ij}+\mathrm{m}(\mathrm{p}) \mathrm{jk}+\mathrm{gm}(\mathrm{p}) \mathrm{ijk}+\varepsilon \mathrm{ijk} \mathrm{l}$, em que yijkl = estimativa dos parâmetros (A ou k); $\mu=$ efeito médio global; gi = efeito do i-ésimo grupo genético $(i=1, \ldots, 4) ; \mathrm{pj}=$ efeito da j-ésima época de nascimento $(\mathrm{j}=1$ :primavera e 2 :verão); $(g p) i j=$ efeito da interação entre grupo genético e época de nascimento; $\mathrm{m}(\mathrm{p}) \mathrm{jk}=$ efeito de suplementação alimentar dentro de época de nascimento; $g m(p) i j k$ = interação entre grupo genético e efeito de suplementação dentro de época de nascimento; e cijkl = erro aleatório. Utilizou-se o procedimento MIXED do SAS (2004), com a opção DDFM=SATTERTH, para calcular o número de graus de liberdade do denominador do quadrado médio residual e obter o valor exato de F.

\section{Resultados e Discussão}

Dos cinco modelos não lineares ajustados, apenas os modelos Brody e Von Bertalanffy não ponderado e ponderado, respectivamente, convergiram para os quatro grupos genéticos. Uma possível explicação é que esses modelos não possuem solução analítica das equações normais, sendo as estimativas dos parâmetros dos modelos não lineares obtidas por algoritmos iterativos.

Ao comparar-se os modelos Brody e Von Bertalanffy, conforme os critérios utilizados de qualidade de ajuste, observa-se, com base nos valores dos coeficientes

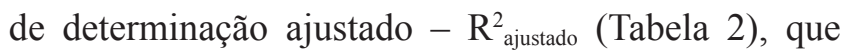
a ponderação permitiu ajuste semelhante para os modelos. Porém, o $\mathrm{R}_{\text {ajustado }}^{2}$ não é um bom diferenciador para a escolha de modelos, independentemente da ponderação, pois todos apresentaram valores altos para esta estatística. Esse resultado confirma os obtidos por Elias (1998), ao analisar dados de peso-idade de vacas zebuínas até a idade adulta, pois, no presente trabalho, verificou-se $\mathrm{R}_{\text {ajustado }}^{2}$ de aproximadamente 0,999 para os modelos não lineares ajustados.

Em relação ao QMR, a ponderação proporcionou redução significativa na variabilidade das estimativas dos QMRs, sendo que o modelo Brody apresentou médias menores. Este resultado discorda do de Beltrán et al. (1992), que encontraram maior QMR para o modelo Brody. No entanto, outros estudos (Brown et al., 1972a, 1972b; Arango \& Van Vleck, 2002) indicam que o modelo Brody é o mais adequado para modelar a curva de crescimento de bovinos, em comparação aos demais modelos, já que os resultados são mais fáceis de serem obtidos e interpretados, apesar de serem menos sensíveis às flutuações no peso. Tanto no modelo Brody quanto no Von Bertalanffy, independentemente do uso da ponderação (Tabela 2), o EPM é negativo, o que indica superestimação dos pesos observados dos grupos genéticos. Esses resultados estão de acordo com os obtidos por Paz et al. (2004), ao utilizar o EPM como critério adicional para verificar a qualidade do ajuste do modelo Logístico não ponderado aos conjuntos de peso-idade de fêmeas CN, AN e SN. No presente trabalho, o modelo Logístico superestimou os pesos, resultado parcialmente discordante do encontrado por Mazzini et al. (2003) que, ao analisar dados de peso-idade de animais da raça Hereford, concluíram que o modelo de Brody não ponderado subestimou a média dos pesos.

Tabela 2. Coeficiente de determinação ajustado $\left(\mathrm{R}_{\text {ajustado }}\right.$ ), quadrado médio do resíduo (QMR), erro de predição médio (EPM) e número de iterações (NI) para os modelos Brody e Von Bertalanffy não ponderados (NP) e ponderados (P), ajustados a dados peso-idade, do nascimento até 100 meses de idade, de fêmeas bovinas de quatro grupos genéticos, nascidas entre 1998 e 2001.

\begin{tabular}{|c|c|c|c|c|c|c|c|c|}
\hline \multirow[t]{2}{*}{ Grupo genético } & \multicolumn{2}{|c|}{$\mathrm{R}_{\text {ajustado }}^{2}$} & \multicolumn{2}{|c|}{ QMR } & \multicolumn{2}{|c|}{ EPM } & \multicolumn{2}{|c|}{ NI } \\
\hline & NP & $\mathrm{P}$ & NP & $\mathrm{P}$ & NP & $\mathrm{P}$ & NP & $\mathrm{P}$ \\
\hline & \multicolumn{8}{|c|}{ Brody } \\
\hline Nelore & 0,871479 & 0,999999 & 2062,9 & 1,0630 & $-2,2338$ & $-1,1848$ & 4 & 15 \\
\hline $1 / 2$ Canchim $+1 / 2$ Nelore & 0,838409 & 0,999934 & 2673,0 & 1,0839 & $-2,9659$ & $-1,2667$ & 6 & 16 \\
\hline $1 / 2$ Angus $+1 / 2$ Nelore & 0,830734 & 0,999931 & 2877,8 & 1,1590 & $-3,7142$ & $-1,2533$ & 4 & 18 \\
\hline \multirow[t]{2}{*}{$1 / 2$ Simental $+1 / 2$ Nelore } & 0,836172 & 0,999940 & 3032,6 & 1,0976 & $-2,8765$ & $-1,1548$ & 5 & 17 \\
\hline & \multicolumn{8}{|c|}{ Von Bertalanffy } \\
\hline Nelore & 0,863969 & 0,999914 & 2183,4 & 1,3723 & $-6,2981$ & $-0,9747$ & 33 & 17 \\
\hline $1 / 2$ Canchim $+1 / 2$ Nelore & 0,829325 & 0,999918 & 2823,3 & 1,3413 & $-6,5420$ & $-1,0255$ & 32 & 17 \\
\hline $1 / 2$ Angus $+1 / 2$ Nelore & 0,81750 & 0,999915 & 3102,8 & 1,4295 & $-7,4838$ & $-1,1061$ & 33 & 17 \\
\hline $1 / 2$ Simental $+1 / 2$ Nelore & 0,825721 & 0,999929 & 3226,0 & 1,3113 & $-6,4780$ & $-0,8597$ & 31 & 17 \\
\hline
\end{tabular}


Os resultados obtidos por Mazzini et al. (2003) e Paz et al. (2004), no ajuste do modelo de Von Bertalanffy, diferem dos encontrados no presente trabalho, em que a ponderação da variância reduziu a superestimação.

A explicação para a discordância entre os resultados encontrados neste trabalho e na literatura discutida acima pode ser atribuído a diversos fatores, tais como tamanho do conjunto de dados, existência de valores influenciais ou "outliers", idade nas últimas pesagens, oscilações nos valores dos pesos com a idade, grupos genéticos diferentes, variações amostrais mendelianas dentro de cada grupo genético, algoritmos usados no processo iterativo e número de pares peso-idade por animal. Quanto à este último, Toral (2008) verificou, por meio de simulação, que, com menos de 49 pesagens por animal, há mais superestimavas ou subestimavas dos parâmetros A e k do modelo de Richards. No presente trabalho, o número mínimo e máximo de pesagens por vacas foram 9 e 35, respectivamente, o que está dentro do intervalo crítico discutido por Toral.

O modelo de Brody ponderado exigiu um maior número de iterações que o de Von Bertalanffy não ponderado; entretanto, esta estatística está relacionada aos valores inicias dos parâmetros (Tabela 2 ).

Com as curvas de crescimento geradas pelas médias dos pesos observados nas diferentes classes de idades e com os pesos estimados a partir dos parâmetros dos modelos, nas mesmas classes de idade, é possível comparar graficamente o ajuste de cada modelo em relação aos pesos observados (Figura 1).

Os modelos Brody e Von Bertalanffy, em geral, ajustaram-se bem aos pesos observados. Contudo, o modelo Brody ponderado apresentou melhor ajuste para todos os animais. O modelo Von Bertalanffy tendeu a superestimar os pesos iniciais para todos os animais dos quatro grupos genéticos e apresentou valores abaixo dos valores médios observados na parte final da curva, o que indica que os pesos foram subestimados nessa fase, como ocorreu em Oliveira et al. (2000).

As maiores estimativas de A foram obtidas para as fêmeas $\mathrm{SN}$, e as menores taxas de maturação $(\mathrm{k})$ para as fêmeas NEL (Tabela 3). Como a idade final foi fixada em 100 meses, os resultados mostram que maiores estimativas de A estão associadas positivamente com maiores valores de $\mathrm{k}$, ou seja, o maior peso adulto e, consequentemente, a maior velocidade de crescimento, foi obtido pelas fêmeas $\mathrm{SN}$, enquanto os menores foram obtidos pelas fêmeas NEL. Caso a idade final seja variável, as vacas com menores valores de $\mathrm{k}$ necessitam de mais tempo para atingir o mesmo peso adulto que as vacas com maiores valores de $\mathrm{k}$.

Ao avaliar as estimativas dos parâmetros dos modelos sem ponderação da variância (Tabela 3), observou-se, para os modelos Brody e Von Bertalanffy, que as vacas $\mathrm{SN}$ apresentaram maior peso assintótico e taxa de maturação, em comparação às vacas NEL, CN e AN. Para o modelo Brody não ponderado, as vacas SN apresentaram estimativas para peso assintótico, em 9,84\% (54,5 kg), 7,02\% (38,9 kg) e 1,79\% (9,9 kg), e taxa de maturação, de $7,66 \%(0,0041$ por mês), $6,72 \%$ ( 0,0036 por mês $)$ e $2,43 \%(0,0013$ por mês $)$, superiores às vacas NEL, $\mathrm{CN}$ e $\mathrm{AN}$, respectivamente.

Quanto ao modelo Von Bertalanffy não ponderado, as vacas $\mathrm{SN}$ apresentaram pesos assintóticos, em 10,26\% $(55,92 \mathrm{~kg}), 7,16 \%(39,05 \mathrm{~kg})$ e $1,74 \%(9,52 \mathrm{~kg})$, e taxa de maturação, de 5,21\% (0,0037 por mês), $6,06 \%$ $(0,0043$ por mês $)$ e $4,65 \%$ ( 0,0033 por mês $)$, superiores às vacas NEL, $\mathrm{CN}$ e $\mathrm{AN}$, respectivamente. Esses resultados divergem dos encontrados na literatura, em que animais mais pesados apresentam menores taxas de maturação, e pode ser atribuído ao viés das estimativas dos parâmetros obtidos sem a correção da heterogeneidade de variância (Pasternak \& Shalev, 1994).

Para as estimativas dos parâmetros estimados por modelos ponderados, observa-se que, embora as vacas SN tenham apresentado valores maiores para peso assintótico $(543,00 ; 523,40 \mathrm{~kg})$, isso não significa necessariamente que são animais precoces, pois as vacas $\mathrm{AN}$ apresentaram maiores médias para a taxa de maturação $(0,0632 ; 0,1063$ por mês $)$ sob os modelos Brody e Von Bertalanffy, sendo as F1 mais precoces que as dos outros grupos genéticos estudados.

Com o modelo Brody, as vacas AN foram 14,71\% ( 0,0093 por mês), $9,01 \%(0,0057$ por mês $)$ e $3,00 \%$ $(0,0019$ por mês) mais precoces que as NEL, CN e $\mathrm{SN}$, respectivamente. Para o modelo Von Bertalanffy, as vacas $\mathrm{F} 1 \mathrm{AN}$ tiveram precocidades superiores, de $14,58 \%$ ( 0,0155 por mês $), 9,40 \%$ ( 0,0100 por mês $)$ e $3,67 \%$ ( 0,0039 por mês $)$, em comparação às NEL, CN e SN, respectivamente. É importante observar que a ponderação proporcionou redução nos erros-padrão das estimativas dos parâmetros (Tabela 3), pois o uso do inverso da variância dos pesos ao longo do tempo atende a um dos requisitos fundamentais do 

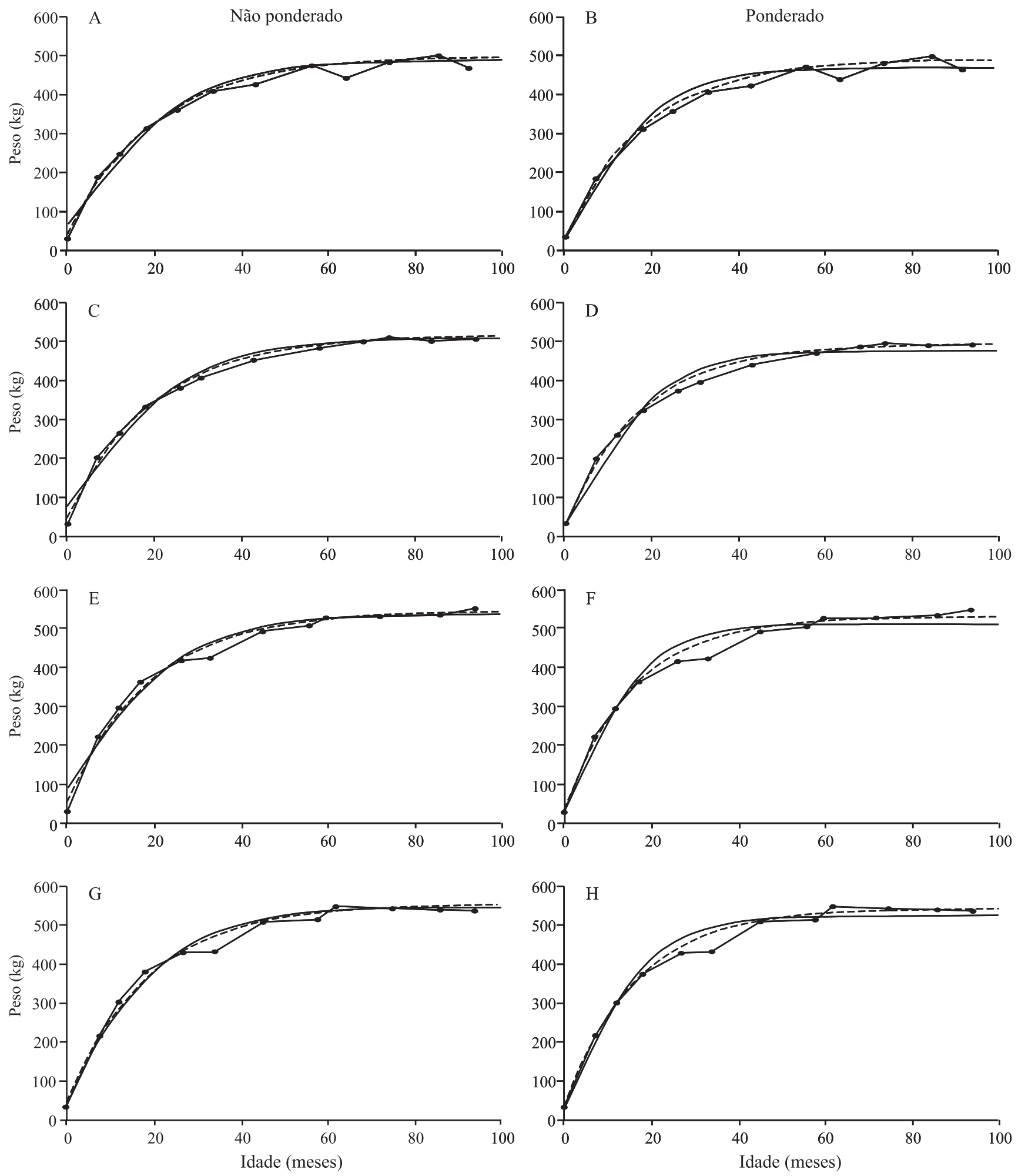

\section{Pesos observados ----Brody — Von Bertalanffy}

Figura 1. Estimativa dos pesos em função da idade, observados e ajustados pelos modelos Brody e Von Bertalanffy não ponderado (A, C, E, G) e ponderado (B, D, F, H), de fêmeas Nelore (A, B), $1 / 2$ Canchim $+1 / 2$ Nelore $(C, D), 1 / 2$ Angus $+1 / 2$ Nelore $(E, F)$ e 1/2Simental +1/2Nelore (G, H) do nascimento até 100 meses de idade, nascidas entre 1998 e 2001. 
uso da metodologia de quadrados mínimos, ou seja, a homogeneidade de variâncias. Segundo Pasternak \& Shalev (1994), há "distúrbio de regressão" quando as variâncias ao longo do tempo não são levadas em consideração. Elias (1998) verificou, ao comparar modelos não lineares de crescimento não ponderado e ponderado pelo inverso da variância dos pesos em diferentes idades de um conjunto completo de dados de peso-idade, do nascimento até a idade adulta de vacas das raças Nelore, Guzerá e Gir, que os modelos não ponderados apresentaram estimativas para os parâmetros $\mathrm{A}$ e $\mathrm{k}$ mais próximas da realidade para cada raça. Para os modelos ponderados, a média de peso assintótico que mais se aproximou do valor observado foi estimada pelo modelo Brody, nas raças Guzerá e Nelore, e pelo modelo de Richards na raça Gir.

Ao observar o formato e o comportamento das curvas de crescimento dos quatro grupos genéticos (Figura 2), observa-se que a curva de crescimento das fêmeas NEL permanece próxima à das fêmeas $\mathrm{CN}$ até aproximadamente 18 meses de idade e, depois, afasta-se gradativamente à medida que se aproxima do valor assintótico. Esse comportamento também pode ser observado com as curvas de crescimento das fêmeas $\mathrm{AN}$ e $\mathrm{SN}$, que apresentam comportamento similar até aproximadamente 24 meses de idade. Independentemente de o modelo ser ponderado ou não ponderado, observa-se que as curvas de crescimento dos grupos genéticos $\mathrm{SN}$ e $\mathrm{AN}$ são similares e superiores às de CN e NEL, tanto para os modelos Brody como Von Bertalanffy. Isto indica que o crescimento varia em relação à idade do animal em função da dieta fornecida e do potencial genético dos animais.

Os modelos Brody e Von Bertalanffy ponderados foram adequados para descrever o crescimento dos animais, sendo o primeiro levemente superior. Portanto, a segunda etapa deste trabalho, em que um modelo misto foi utilizado para verificar a influência dos efeitos ambientais e genéticos sobre as estimativas dos parâmetros do modelo não linear, foi realizada apenas com o modelo Brody ponderado.

$\mathrm{O}$ peso assintótico e a taxa de maturação foram afetados significativamente ( $<<0,01$, Tabela 4) pelos efeitos de grupo genético, época de nascimento e suplementação alimentar.

As vacas que nasceram durante o outono, época das secas, foram mais pesadas e apresentaram taxa de maturidade mais elevada. Esses resultados são condizentes com os encontrados por Silva et al. (2004), ao observar efeito significativo de época de nascimento sobre a taxa de crescimento da desmama a um ano de idade e a taxa de crescimento desde um ano até ao sobreano, em que os animais nascidos no período seco apresentaram maiores taxas de crescimento do que os animais que nasceram no período chuvoso. O efeito de época de nascimento é uma importante fonte de variação, pois ocorrem flutuações na quantidade e na qualidade de pastagens, ao longo da vida do animal, e no manejo dos animais, em decorrência das flutuações de clima e de ambiente. Os animais mais jovens, ainda dependentes em grande parte da alimentação materna, sofrem as consequências dessas modificações de forma indireta,

Tabela 3. Estimativas do peso assintótico (A), constante de integração (b), taxa de maturação (k) e respectivos erros-padrão para os modelos Brody e Von Bertalanffy não ponderados e ponderados, ajustados a dados peso-idade, do nascimento até 100 meses de idade, de fêmeas bovinas nascidas entre 1998 e 2001.

\begin{tabular}{|c|c|c|c|c|c|c|}
\hline \multirow[t]{2}{*}{ Grupo genético } & \multicolumn{2}{|c|}{ A } & \multicolumn{2}{|c|}{$\mathrm{b}$} & \multicolumn{2}{|c|}{$\mathrm{k}$} \\
\hline & NP & $\mathrm{P}$ & NP & $\mathrm{P}$ & NP & $\mathrm{P}$ \\
\hline & \multicolumn{6}{|c|}{ Brody } \\
\hline Nelore & $498,97 \pm 2,2026$ & $493,27 \pm 2,0224$ & $0,9259 \pm 0,0085$ & $0,9405 \pm 0,0008$ & $0,0494 \pm 0,0009$ & $0,0539 \pm 0,0006$ \\
\hline $1 / 2$ Canchim $+1 / 2$ Nelore & $514,57 \pm 2,1675$ & $504,45 \pm 1,9863$ & $0,9112 \pm 0,0093$ & $0,9377 \pm 0,0008$ & $0,0499 \pm 0,0010$ & $0,0575 \pm 0,0006$ \\
\hline $1 / 2$ Angus $+1 / 2$ Nelore & $543,56 \pm 2,3315$ & $529,64 \pm 2,0914$ & $0,8976 \pm 0,0100$ & $0,9386 \pm 0,0009$ & $0,0522 \pm 0,0011$ & $0,0632 \pm 0,0008$ \\
\hline \multirow[t]{2}{*}{$1 / 2$ Simental $+1 / 2$ Nelore } & $553,47 \pm 2,4550$ & $543,00 \pm 2,2963$ & $0,9119 \pm 0,0104$ & $0,9374 \pm 0,0009$ & $0,0535 \pm 0,0011$ & $0,0613 \pm 0,0008$ \\
\hline & \multicolumn{6}{|c|}{ Von Bertalanffy } \\
\hline Nelore & $488,94 \pm 1,9535$ & $472,87 \pm 1,8525$ & $0,4898 \pm 0,0092$ & $0,5984 \pm 0,0019$ & $0,0672 \pm 0,0014$ & $0,0908 \pm 0,0009$ \\
\hline $1 / 2$ Canchim $+1 / 2$ Nelore & $505,81 \pm 1,9450$ & $485,87 \pm 1,8225$ & $0,4704 \pm 0,0095$ & $0,5942 \pm 0,0019$ & $0,0666 \pm 0,0015$ & $0,0963 \pm 0,0010$ \\
\hline $1 / 2$ Angus $+1 / 2$ Nelore & $535,35 \pm 2,1542$ & $510,51 \pm 1,9343$ & $0,4506 \pm 0,0098$ & $0,5969 \pm 0,0021$ & $0,0679 \pm 0,0016$ & $0,1063 \pm 0,0012$ \\
\hline $1 / 2$ Simental $+1 / 2$ Nelore & $544,86 \pm 2,2379$ & $523,40 \pm 2,0461$ & $0,4708 \pm 0,0108$ & $0,5948 \pm 0,0020$ & $0,0709 \pm 0,0017$ & $0,1024 \pm 0,0012$ \\
\hline
\end{tabular}

Pesq. agropec. bras., Brasília, v.46, n.3, p.262-271, mar. 2011 

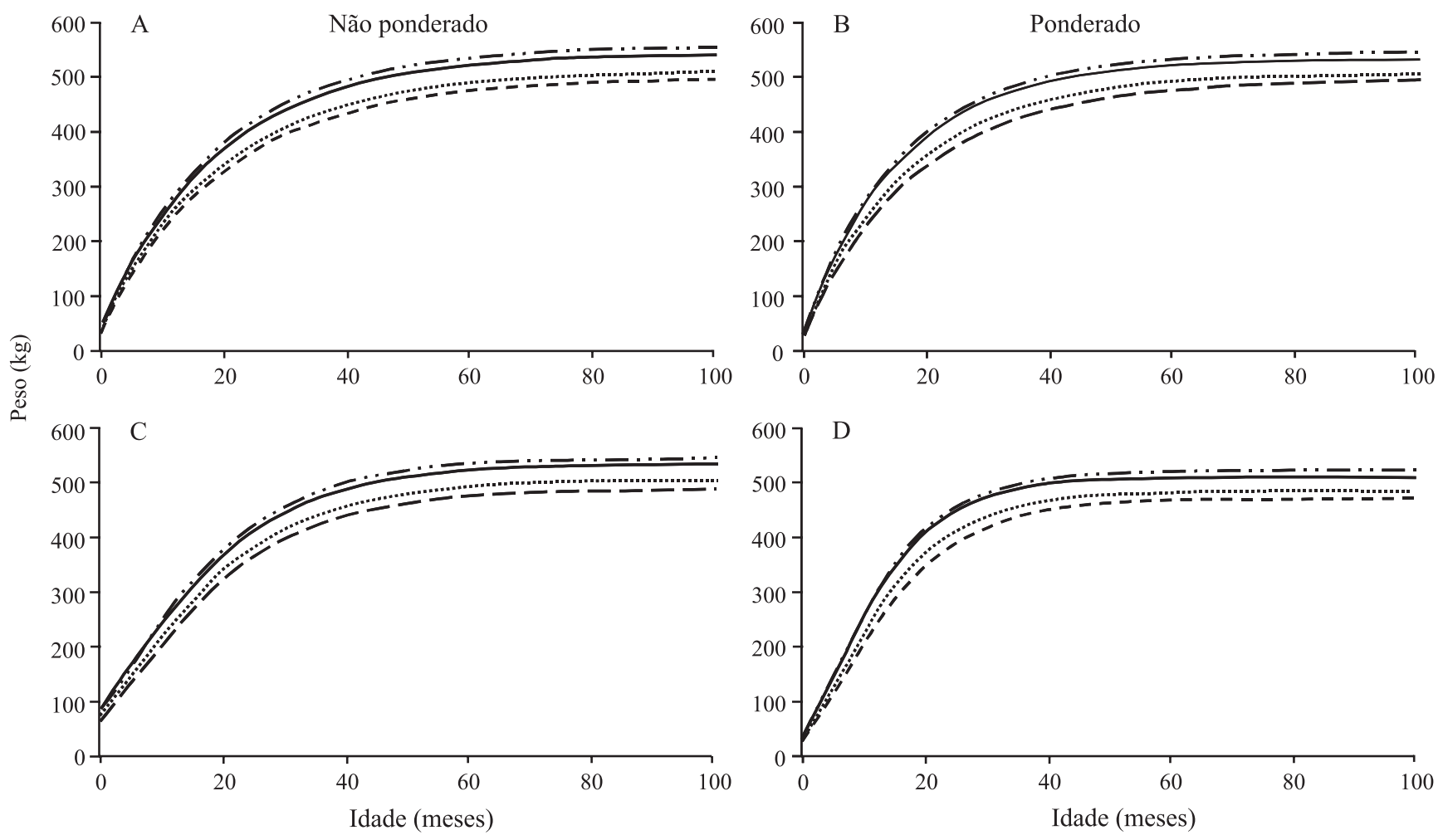

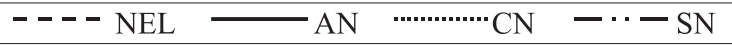

Figura 2. Curvas de crescimento estimadas pelos modelos Brody (A, B) e Von Bertalanffy (C, D) não ponderado (A, C) e ponderado $(B, D)$ para fêmeas dos grupos genéticos Nelore (NEL), $1 / 2$ Canchim $+1 / 2$ Nelore $(C N), 1 / 2$ Angus $+1 / 2$ Nelore (AN) e $1 / 2$ Simental $+1 / 2$ Nelore (SN) do nascimento até 100 meses de idade, nascidas entre 1998 e 2001.

Tabela 4. Médias ajustadas e erros-padrão das estimativas do peso assintótico (A) e taxa de maturação (k) obtidos do modelo Brody ponderado para época de nascimento (outono ou primavera), suplementação alimentar $(0,1,5$ e 3,0 kg por animal por dia) e grupos genéticos. Foram utilizados dados peso-idade, do nascimento até 100 meses de idade, de fêmeas bovinas, nascidas entre 1998 e $2001^{(1)}$.

\begin{tabular}{|c|c|c|c|c|c|}
\hline \multirow[t]{2}{*}{ Grupo genético } & \multicolumn{2}{|c|}{ Outono } & \multicolumn{2}{|c|}{ Primavera } & \multirow[t]{2}{*}{ Média } \\
\hline & 0 & 3,0 & 1,5 & 3,0 & \\
\hline \multicolumn{6}{|c|}{ Peso assintótico (A) } \\
\hline Nelore & $504,3 \pm 9,50$ & $506,1 \pm 9,90$ & $496,8 \pm 10,65$ & $479,6 \pm 10,13$ & $496,7 \pm 5,02 \mathrm{C}$ \\
\hline $1 / 2$ Canchim $+1 / 2$ Nelore & $526,1 \pm 9,90$ & $516,9 \pm 10,13$ & $478,1 \pm 9,90$ & $493,9 \pm 9,90$ & $503,8 \pm 4,97 \mathrm{C}$ \\
\hline $1 / 2$ Angus $+1 / 2$ Nelore & $540,6 \pm 10,94$ & $550,0 \pm 9,48$ & $532,3 \pm 11,26$ & $499,2 \pm 12,41$ & $530,5 \pm 5,53 \mathrm{~B}$ \\
\hline $1 / 2$ Simental $+1 / 2$ Nelore & $544,4 \pm 10,38$ & $568,4 \pm 9,90$ & $539,9 \pm 13,40$ & $521,0 \pm 11,61$ & $543,4 \pm 5,70 \mathrm{~A}$ \\
\hline \multirow[t]{2}{*}{ Média } & $528,8 \pm 5,10$ & $535,3 \pm 4,93$ & $511,8 \pm 5,69$ & $498,4 \pm 5,53$ & \\
\hline & \multicolumn{2}{|c|}{$532,1 \pm 3,54 \mathrm{a}$} & \multicolumn{2}{|c|}{$505,1 \pm 3,96 \mathrm{~b}$} & \\
\hline \multicolumn{6}{|c|}{ Taxa de maturação (k) } \\
\hline Nelore & $0,050 \pm 0,0018$ & $0,056 \pm 0,0019$ & $0,056 \pm 0,0021$ & $0,057 \pm 0,0020$ & $0,055 \pm 0,0009 \mathrm{C}$ \\
\hline $1 / 2$ Canchim $+1 / 2$ Nelore & $0,056 \pm 0,0019$ & $0,067 \pm 0,0020$ & $0,059 \pm 0,0019$ & $0,058 \pm 0,0019$ & $0,060 \pm 0,0009 \mathrm{~B}$ \\
\hline $1 / 2$ Angus $+1 / 2$ Nelore & $0,062 \pm 0,0021$ & $0,068 \pm 0,0018$ & $0,059 \pm 0,0022$ & $0,067 \pm 0,0024$ & $0,064 \pm 0,0010 \mathrm{~A}$ \\
\hline $1 / 2$ Simental $+1 / 2$ Nelore & $0,062 \pm 0,0020$ & $0,066 \pm 0,0019$ & $0,057 \pm 0,0026$ & $0,061 \pm 0,0022$ & $0,062 \pm 0,0011 \mathrm{AB}$ \\
\hline \multirow[t]{2}{*}{ Média } & $0,057 \pm 0,0010 \mathrm{c}$ & $0,065 \pm 0,0009 \mathrm{a}$ & $0,058 \pm 0,0011 \mathrm{cb}$ & $0,061 \pm 0,0010 \mathrm{abc}$ & \\
\hline & \multicolumn{2}{|c|}{$0,061 \pm 0,0007$} & \multicolumn{2}{|c|}{$0,059 \pm 0,0007$} & \\
\hline
\end{tabular}

${ }^{(1)}$ Médias seguidas de letras iguais, minúsculas nas linhas e maiúsculas nas colunas, não diferem entre si pelo teste t de Student, a $5 \%$ de probabilidade. 
pelo efeito sobre a produção de leite, e direta, pela redução de dieta sólida de qualidade em uma fase de grande exigência nutricional; as variações ambientais que ocorrem de ano para ano também têm reflexos diretos sobre o desempenho dos animais (Silva, 1990). Neste trabalho, o efeito de época de nascimento confunde-se com o efeito de níveis de suplementação, já que, no outono, os animais dos diferentes grupos genéticos receberam 0 e $3,0 \mathrm{~kg}$ de concentrado por animal por dia, enquanto, na primavera, receberam 1,5 e $3,0 \mathrm{~kg}$ de concentrado por animal por dia. Rocha et al. (2003), ao trabalhar com vacas Nelore adultas, relataram que as vacas nascidas no período chuvoso foram menores que as nascidas na época da seca. Os autores atribuíram essa diferença ao ambiente mais propício encontrado pelos animais nascidos na seca, durante os primeiros meses de vida. Entretanto, esse resultado discorda dos relatados por Brown et al. (1972a), ao observar que a estação de nascimento não foi fonte de variação importante para os parâmetros A e k em animais Angus e Hereford; a variação em A, para Angus e Hereford, foi inexplicada em ambas as raças.

O efeito de grupo genético foi significativo $(p<0,01)$ para $\mathrm{A}$, o que já era esperado. A suplementação alimentar para o efeito $\mathrm{k}$ também apresentou resultado significativo $(p<0,01)$. Independentemente do grupo genético, as fêmeas que receberam a maior suplementação alimentar em ambas as estações de nascimento, tiveram o maior crescimento até 100 meses de idade. Contudo, a suplementação não influenciou os resultados das estimativas do parâmetro $\mathrm{A}$, o que pode estar associado aos efeitos compensatórios durante o crescimento dos animais, tais como variações sazonais com consequência na quantidade e na qualidade de alimento disponível, que podem minimizar ou anular vantagens na velocidade de crescimento, em virtude do efeito da suplementação alimentar.

\section{Conclusões}

1. Os modelos Brody e Von Bertalanffy são adequados para ajustar as curvas de crescimento, do nascimento até a idade adulta, de vacas de diferentes grupos genéticos.

2. A heterogeneidade de variância dos pesos observados ao longo das idades deve ser considerada na estimação dos parâmetros.
3. A forma e a variação das curvas de crescimento, do nascimento até a idade adulta, de vacas de diferentes grupos genéticos, dependem do componente genético, da época de nascimento dos animais e das condições de manejo alimentar.

4. As curvas de crescimento ajustadas para as vacas dos grupos genéticos $1 / 2$ Simental $+1 / 2$ Nelore e $1 / 2$ Angus $+1 / 2$ Nelore são superiores assintoticamente às das vacas dos grupos genéticos $1 / 2$ Canchim $+1 / 2$ Nelore e Nelore.

\section{Agradecimentos}

À Coordenação de Aperfeiçoamento de Pessoal de Nível Superior e à Fundação de Amparo à Pesquisa do Estado de São Paulo, pelo apoio financeiro; à Embrapa Pecuária Sudeste, pela disponibilidade dos dados.

\section{Referências}

ARANGO, J.A.; VAN VLECK, L.D. Size of beef cows: early ideas, new developments. Genetic and Molecular Research, v.50, p.51-63, 2002.

BELTRÁN, J.J.; BUTTS JUNIOR, W.T.; OLSON, T.A.; KOGER, M. Growth patterns of two lines of Angus cattle selected using predicted growth parameters. Journal of Animal Science, v.70, p.734-741, 1992.

BROWN, J.E.; BROWN, C.J.; BUTTS, W.T. A discussion of the genetic aspects of weight, mature weight and rate of maturing in Hereford and Angus cattle. Journal of Animal Science, v.34, p.525-537, 1972a.

BROWN, J.E.; BROWN, C.J.; BUTTS, W.T. Relationships among weights, gains and earliness of maturing in Hereford and Angus females. Journal of Animal Science, v.35, p.507-517, 1972 b.

BROWN, J.E.; FITZHUGH JUNIOR, H.A.; CARTWRIGTH, T.C. A comparison of nonlinear models for describing weight-age relationships in cattle. Journal of Animal Science, v.42, p.810-818, 1976.

ELIAS, A.M. Análise de curvas de crescimento de vacas Nelore, Guzerá e Gir. 1998. 128p. Dissertação (Mestrado) - Escola Superior de Agricultura Luiz de Queiroz, Piracicaba.

FORNI, S.; PILES, M.; BLASCO, A.; VARONA, L.; OLIVEIRA, H.N.; LÔBO, R.B.; ALBUQUERQUE, L.G. Analysis of beef cattle longitudinal data applying a nonlinear model. Journal of Animal Science, v.85, p.3189-3197, 2007.

FREITAS, A. de R. Curvas de crescimento na produção animal. Revista Brasileira de Zootecnia, v.34, p.786-795, 2005.

GUEDES, M.H.P.; MUNIZ, J.A.; PEREZ, J.R.O.; SILVA, F.F. e; AQUINO, L.H. de; SANTOS, C.L. dos. Estudo das curvas de crescimento de cordeiros das raças Santa Inês e Bergamácia 
considerando Heterogeneidade de variâncias. Ciência e Agrotecnologia, v.28, p.381-388, 2004.

HARTLEY, H.O. The modified Gauss-Newton method for the fitting of non-linear regression functions by least squares. Thechnometrics, v.3, p.269-280, 1961.

MAZZINI, A.R. de A.; MUNIZ, J.A.; AQUINO, L.H. de; SILVA, F.F. e. Análise da curva de crescimento de machos Hereford. Ciência e Agrotecnologia, v.27, p.1105-1112, 2003.

MAZZINI, A.R. de A.; MUNIZ, J.A.; SILVA, F.F. e; AQUINO, L.H. de. Curva de crescimento de novilhos Hereford: heterocedasticidade e resíduos autorregressivos. Ciência Rural, v.35, p.422-427, 2005.

MENDES, P.N.; MUNIZ, J.A.; SILVA, F.F. e; MAZZINI, A.R. de A. Modelo logístico difásico no estudo do crescimento de fêmeas da raça Hereford. Ciência Rural, v.38, p.1984-1990, 2008.

NOBRE, P.R.C.; ROSA, A. do N.; SILVA, L.O.C. da; EVANGELISTA, S.R.M. Curvas de crescimento de gado Nelore ajustadas para diferentes freqüências de pesagens. Pesquisa Agropecuária Brasileira, v.22, p.1027-1037, 1987.

OLIVEIRA, H.N. de; LÔBO, R.B.; PEREIRA, C.S. Comparação de modelos não-lineares para descrever o crescimento de fêmeas da Raça Guzerá. Pesquisa Agropecuária Brasileira, v.35, p.1843-1851, 2000.

PASTERNAK, H.; SHALEV, B.A. The effect of a feature of regression disturbance on the efficiency of fitting growth curves. Growth, Development and Aging, v.58, p.33-39, 1994.

PAZ, C.C.P. de; PACKER I.U.; FREITAS, A.R. de; TAMBASCO-TALHARI, D.; REGITANO, L.C. de A.; ALENCAR, M.M. de; G.M. da. Ajuste de modelos não-lineares em estudos de associação entre polimorfismos genéticos e crescimento em bovinos de corte. Revista Brasileira de Zootecnia, v.33, p.1416-1425, 2004.

PEROTTO, D.; CUE, R.I.; LEE, A.J. Comparison of nonlinear functions for describing the growth curve of three genotypes of dairy cattle. Canadian Journal of Animal Science, v.72, p.773-782, 1992.
ROCHA, E.D.; ANDRADE, V.J.; EUCLIDES FILHO, K.; NOGUEIRA, E.; FIGUEIREDO, G.R. Tamanho de vacas Nelore adultas e seus efeitos no sistema de produção de gado de corte. Arquivo Brasileiro de Medicina Veterinária e Zootecnia, v.55, p.474-479, 2003.

RODRIGUES, A. de A.; CRUZ, G.M. de; ALENCAR, M.M. de; BARBOSA, P.F.; CORRÊA, L. de A. Efeito de nível nutricional na época da seca no ganho de peso, idade e peso ao primeiro cio de novilhas de diferentes grupos genéticos. In: REUNIÃO ANUAL DA SOCIEDADE BRASILEIRA DE ZOOTECNIA, 41., 2004, Campo Grande. Anais. Campo Grande: Sociedade Brasileira de Zootecnia, 2004. 1 CD-ROM.

SAS INSTITUTE. SAS/Stat user's guide. Version 9.1. Cary: SAS Institute, 2004.

SILVA, L.O.C. da. Tendência genética e interação genótipo $x$ ambiente em rebanhos Nelore, criados a pasto no Brasil Central. 1990. 113p. Tese (Doutorado) - Universidade Federal de Viçosa, Viçosa.

SILVA, N.A.M. da; AQUINO, L.H. de; SILVA, F.F. e; OLIVEIRA, A.I.G. de. Curvas de crescimento e influência de fatores não-genéticos sobre as taxas de crescimento de bovinos da raça Nelore. Ciência e Agrotecnologia, v.28, p.647-654, 2004.

SOUZA, G. da S. Introdução aos modelos de regressão linear e não-linear. Brasília: Embrapa-SPI: Embrapa-seA, 1998. 489 p.

TEDESCHI, L.O.; BOIN, C.; NARDON, R.F.; LEME, P.R. Estudo da curva de crescimento de animais da raça Guzerá e seus cruzamentos alimentados a pasto, com e sem suplementação. 1. Análise e seleção das funções não-lineares. Revista Brasileira de Zootecnia, v.29, p.630-637, 2000.

TORAL, F.L.B. Número e intervalo de pesagens para estimação de parâmetros de curvas de crescimento em bovinos. Revista Brasileira de Zootecnia, v.37, p.2120-2128, 2008.

Recebido em 4 de setembro de 2010 e aprovado em 28 de fevereiro de 2011 